\title{
Determinants of the efficacy of HIV latency-reversing agents and implications for drug and treatment design
}

\author{
Ruian Ke, ${ }^{1,2}$ Jessica M. Conway, ${ }^{3}$ David M. Margolis,, ${ }^{4,5,6}$ and Alan S. Perelson ${ }^{2}$ \\ 'Department of Mathematics, North Carolina State University, Raleigh, North Carolina, USA. ${ }^{2}$ Theoretical Biology \\ and Biophysics Group, MS-K710, Los Alamos National Laboratory, Los Alamos, New Mexico, USA. ${ }^{3}$ Department of \\ Mathematics and Center for Infectious Disease Dynamics, Pennsylvania State University, State College, Pennsylvania, \\ USA. ${ }^{4}$ University of North Carolina (UNC) HIV Cure Center, UNC Institute of Global Health and Infectious Diseases, \\ ${ }^{5}$ Departments of Medicine, Microbiology and Immunology, UNC Chapel Hill School of Medicine, and ${ }^{6}$ Department of \\ Epidemiology, UNC Chapel Hill School of Public Health, University of North Carolina at Chapel Hill, Chapel Hill, \\ North Carolina, USA.
}

HIV eradication studies have focused on developing latency-reversing agents (LRAs). However, it is not understood how the rate of latent reservoir reduction is affected by different steps in the process of latency reversal. Furthermore, as current LRAs are host-directed, LRA treatment is likely to be intermittent to avoid host toxicities. Few careful studies of the serial effects of pulsatile LRA treatment have yet been done. This lack of clarity makes it difficult to evaluate the efficacy of candidate LRAs or predict long-term treatment outcomes. We constructed a mathematical model that describes the dynamics of latently infected cells under LRA treatment. Model analysis showed that, in addition to increasing the immune recognition and clearance of infected cells, the duration of HIV antigen expression (i.e., the period of vulnerability) plays an important role in determining the efficacy of LRAs, especially if effective clearance is achieved. Patients may benefit from pulsatile LRA exposures compared with continuous LRA exposures if the period of vulnerability is long and the clearance rate is high, both in the presence and absence of an LRA. Overall, the model framework serves as a useful tool to evaluate the efficacy and the rational design of LRAs and combination strategies.

Conflict of interest: DMM reports consultancy for Merck and ASP for Cilead, Vir Biotechnology, and Arbutus Biopharma. DMM also holds common stock in Gilead and ASP in Merck.

License: Copyright 2018, American Society for Clinical Investigation.

Submitted: June 27, 2018

Accepted: August 30, 2018

Published: October 18, 2018

\section{Reference information:}

JCI Insight. 2018;3(20):e123052.

https://doi.org/10.1172/jici.

insight.123052.

\section{Introduction}

Current treatment for human immunodeficiency virus (HIV) infection employs combination antiretroviral therapy (cART), which effectively suppresses HIV replication, reduces viremia to levels below the limit of detection of clinical assays, and substantially reduces morbidity and mortality of HIV-infected patients (1). However, cART does not eradicate HIV and consequently lifelong ART is required. One important mechanism that allows HIV to persist in $\mathrm{HIV}^{+}$individuals in spite of treatment with highly potent cART is the existence and maintenance of a stable population of long-lived latently infected cells, i.e., the HIV latent reservoir $(2,3)$. Recently, extensive efforts have focused on developing novel therapeutics to purge the reservoir, and ultimately cure HIV infection (4-8). One promising strategy involves treating patients with a class of drugs, termed latency-reversing agents (LRAs), which induce HIV gene expression in latently infected cells. Although cells that express virus or antigen might then die due to viral cytopathic effects, current strategies now seek to employ immune-mediated clearance mechanisms to eliminate infected cells. A range of LRAs have been proposed recently (9). They include histone deacetylase inhibitors (HDACis) such as vorinostat (10-13), romidepsin (14), and panobinostat (15), histone methyltransferases inhibitors (16), the anti-alcoholism drug disulfiram $(17,18)$, protein kinase $C$ agonists such as bryostatin-1 (19), and bromodomain inhibitors such as JQ1 (20). Several LRAs have already been tested in clinical trials, and trials for other LRAs with a variety of drugs/therapeutic vaccines are underway $(6,7,9)$. The availability of a large number of LRA candidates raises important questions about how to evaluate and compare candidate LRAs prior to in vivo testing, rationally select promising LRA dosing strategies or LRA combinations for in vivo testing, and determine which characteristics of LRA performance must be improved. 
Currently, studies have focused on the impact of LRAs on latency reversal or clearance processes (10-13, $15,18,21,22)$. Assessment of this is primarily based on experimental determination of the extent of increase in markers of HIV gene expression, such as cell-associated unspliced (CA-US) HIV RNA (10, 12, $13,21)$ and intracellular HIV proteins (23). Other methods to assess LRA effectiveness include measuring the number of cells with integrated HIV DNA or the size of the replication-competent reservoir using viral outgrowth assays (24-28). However, latency reversal is a dynamic process potentially affected by many processes, including activation of HIV gene expression and subsequent expression of HIV antigen, clearance of induced cells, reestablishment of latency after HIV expression (22), and cell proliferation (28-30). Therefore, a quantitative framework that might integrate these processes is needed to formally evaluate the efficacy of candidate LRAs and improve the design of further studies $(31,32)$.

Mathematical modeling has been used to understand the dynamics of the HIV latent reservoir under cART and LRAs (31-35) as well as HIV rebound that results from activation of latently infected cells after cessation of ART (36). In particular, one recent modeling study on LRAs pointed out that the impact of two LRAs may be different even when experimental measurements of HIV expression yield similar readouts (32). Here, we develop a mathematical model specifically focusing on each step of latency reversal under LRA therapy and how the rate of processes in each step determines LRA efficacy. It is likely that a pulsatile LRA exposure is needed to minimize the side effects of an LRA, as in previous clinical trials for panobinostat (15) and vorinostat (11). We thus derive general principles of how LRA efficacy may depend on the dynamics of latency reversal and LRA exposure patterns.

\section{Results}

Definition of LRA efficacy. First, a measure for LRA efficacy is needed. Previously, mathematical models have provided the framework to estimate the efficacy of cART (37-40). cART inhibits or disrupts steps in the HIV viral life cycle and therefore efficacy is estimated in terms of the drug-induced reduction in the rate of the targeted life-cycle step. For example, the efficacy of a protease inhibitor can be defined by the reduction in the rate of infectious virion production (41), whereas the efficacy of an integrase inhibitor can be defined by the reduction in the rate of proviral integration (42). However, LRAs act by inducing HIV gene expression in latently infected cells such that those cells can be recognized and killed/cleared by viral cytopathic effects or host immune responses. Thus, the definition of efficacy for cART does not apply to LRAs. Given that the overall goal of LRAs is to reduce the size of the HIV latent reservoir, here we measure the efficacy of LRAs as the reduction of the size of the reservoir after a certain period of time (e.g., treatment and follow-up periods used in clinical trials) and use this definition to evaluate the impact of LRAs.

$A$ model for latency reversal with an LRA. To understand the latency-reversing process under LRAs, we first developed a model using ordinary differential equations (ODEs; see Methods) based on previous models $(31,33,43)$. The model incorporates several key steps in the latency reversal process under an LRA (Figure 1). First, we consider the induction of HIV gene expression and subsequent HIV antigen expression. The rate of this step is described by parameter $\alpha$, the latency reversal (LR) rate in the model. Second, we consider recognition and clearance of these now vulnerable infected cells by the immune system or viral cytopathic effects (although current LRAs seem to be insufficient to induce a high level of viral production). The rate of this step is described by the parameter $\delta$, the clearance rate in the model. Third, we consider an LRA refractory period before latency reestablishment. Induced cells can reestablish latency because activation of HIV expression by an LRA is not permanent. If induced cells are not cleared, over time viral and cellular factors may become insufficient to support HIV expression, leading to reestablishment of latency $(22,31)$. In addition, studies using the LRA vorinostat showed that a population of cells exposed to this LRA go through a period during which they become relatively unresponsive to a subsequent exposure to vorinostat $(10,11)$. This may be due to downregulation of positive regulators of transcription, such as histone acetyl transferases, and/or transcriptional repression through upregulation of trimethylation at transcription start sites $(44,45)$. Here, without losing generality, we include this refractory period in the model, although this period can be set to 0 for LRAs that do not induce a refractory period. The rates of the transition of cells from an induced state to a refractory state and then to latency reestablishment are described by the parameter $\gamma$ (the exit rate), and the parameter $\omega$ (the latency re-establishment rate) in the model, respectively. Note that $1 / \gamma$ is the period of vulnerability (for immune killing) and $1 / \omega$ is the refractory period of latently infected cells. 

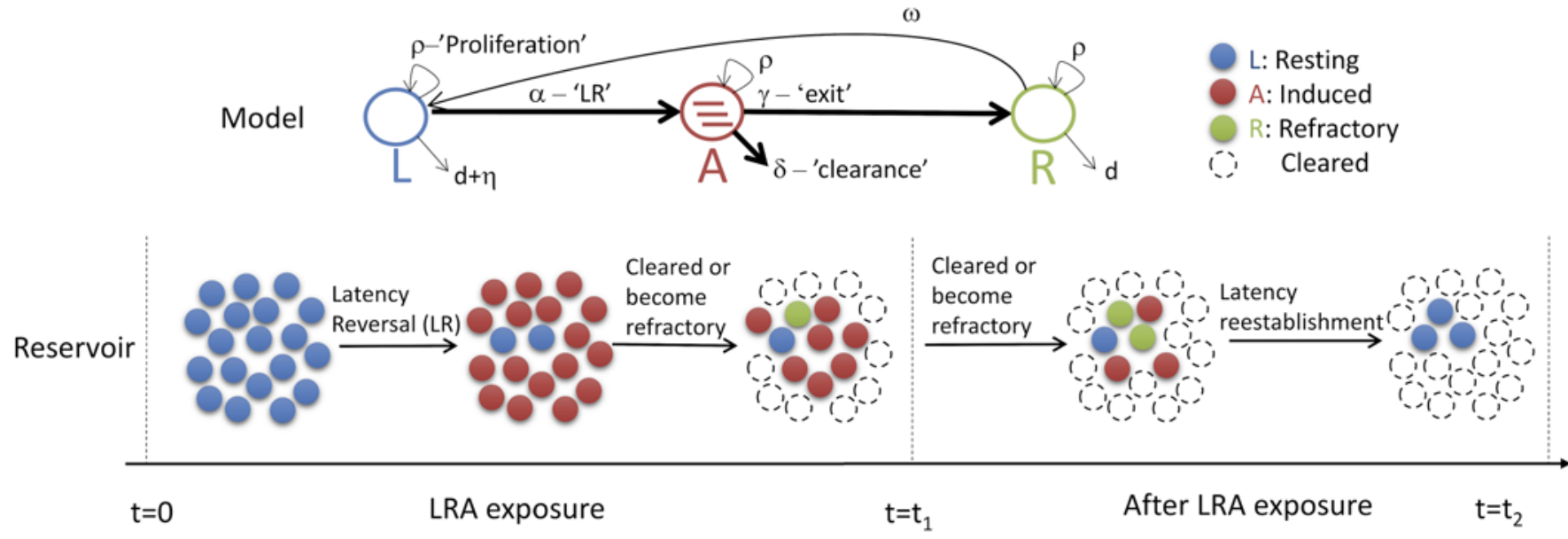

Figure 1. Diagram of a compartmental model for the dynamics of the HIV latent reservoir on and after latency reversing agent (LRA) exposure. In the absence of an LRA, the unactivated (resting) latently infected cell population ( $L$ and blue dots) is affected by cell proliferation, natural cell death, and natural cell activation (at per capita rates, $\rho, d$, and $\eta$, respectively). Upon LRA exposure, latently infected cells become induced ( $A$ and red dots) at per capita rate $\alpha$ (i.e., the latency reversal [LR] rate). Over time, the induced cells either are killed/cleared by immune effector cells at per capita rate $\delta$ (i.e., the clearance rate) or lose induction and become refractory to further immediate latency reversal ( $R$ and green dots) at per capita rate $\gamma$ (the exit rate). Cells in a refractory state return to the latent but responsive state $(L)$ at rate $\omega$. After LRA exposure, if induced cells stay in the induced state, e.g., HIV antigen is continuously expressed on the cell surface, induced cells can still be cleared by immune effector cells; otherwise, they become refractory to LRA and eventually return to an unactivated state. A second cycle of LRA treatment may then proceed, leading to further reduction in the reservoir size. All latently infected cells proliferate at per capita rate $\rho$.

Determinants of LRA efficacy - analytical approximation. In order to understand how the LRA efficacy depends on the key parameter values in our model, we first derived analytical approximations to the longterm dynamics of the latent reservoir in the presence of LRAs (see supplemental text; supplemental material available online with this article; https://doi.org/10.1172/jci.insight.123052DS1). The model (equation II in Methods) predicts that the total size of the reservoir decreases exponentially in the long term. To intuitively understand the overall effect of an LRA, we further make the simplifying assumption that the induced cells $(A)$ go to the uninduced state $(L)$ directly without going through the refractory state. The exponential decrease rate $(\Psi)$ that can then be approximated as shown in equation I:

$$
\Psi=\frac{1}{2}\left(\alpha+\gamma+\delta-\sqrt{(\alpha+\gamma+\delta)^{2}-4 \alpha \delta}\right)-\rho
$$

The value of $\Psi$ then serves as a measure of LRA efficacy under LRA exposure. A few simple rules can be derived from the expression for $\Psi$ : (a) $\alpha$ and $\delta$ always appears together as sums or products. This suggests that the LR rate $\alpha$ and the clearance rate $\delta$ are equally important in determining LRA efficacy. (b) When the deactivation rate $\gamma$ is very small compared with $\alpha+\delta, \gamma$ can be neglected in the $\alpha+\gamma+\delta$ term, i.e., $\alpha+\gamma+\delta$ $\approx \alpha+\delta$. Then, the following simple expressions for $\Psi$ can be derived: $\Psi \approx \alpha-\rho$, when $\alpha<\delta$, and $\Psi \approx \delta-\rho$, when $\alpha>\delta$. This suggests that the efficacy of an LRA is mostly driven by the slower of the 2 rates $\alpha$ and $\delta$ when the deactivation rate $\gamma$ is small.

When the sum of $\alpha$ and $\delta$ (i.e., $\alpha+\delta$ ) is kept a constant (e.g., in cases where there exists a trade-off between optimizing the LR rate and the clearance rate), maximizing the product of $\alpha$ and $\delta$ always maximizes $\Psi$.

The strong dependence of $\Psi$ on the proliferation rate $\rho$ suggests that changes in this rate during LRA interventions would have major impact on the efficacy of the interventions.

Determinants of LRA efficacy - simulation of clinical trials. We then performed model simulations to assess the impact of LRA therapy on the size of reservoir reduction under a clinical setting where an LRA is administered in cycles (11). We first performed simulations for one LRA dosing cycle, where we assume a patient is on an LRA for 1 day and off an LRA for 2 days (as in previous clinical trials; see ref. 11). In our previous work, we estimated that for vorinostat, the LR rate is in general high (1.8/day on average); however, the induced cell clearance rate is very low $(<0.1 /$ day $)(31)$. With these parameter values, the reservoir reduction is small, approximately $5 \%$ (Figure $2 \mathrm{~A}$ ). If the induced cell clearance rate 
A

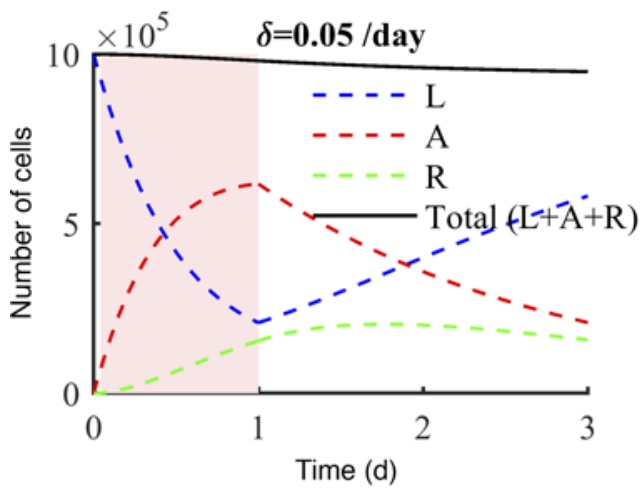

One LRA dosing cycle

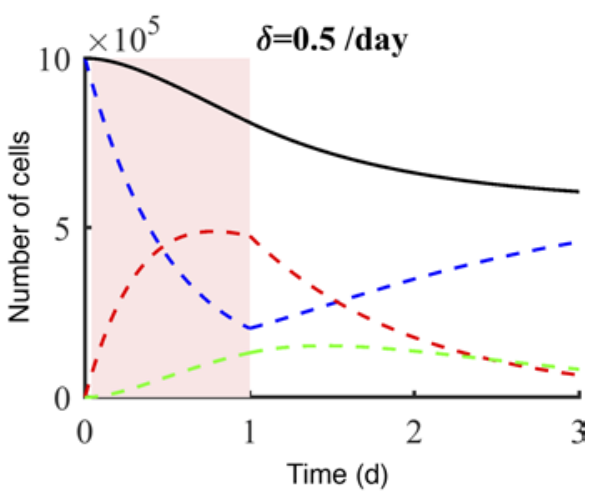

B

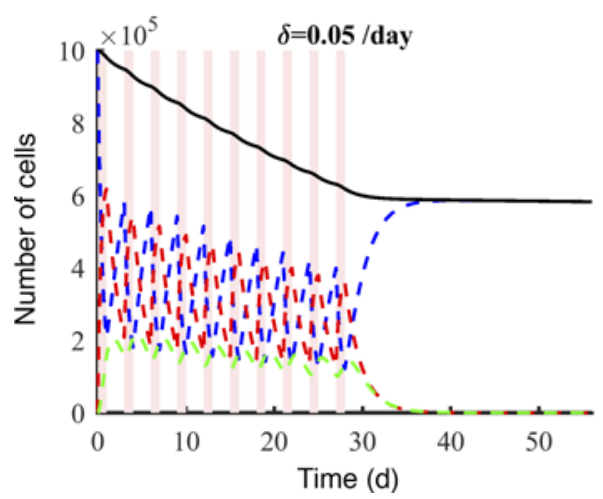

Ten LRA dosing cycles

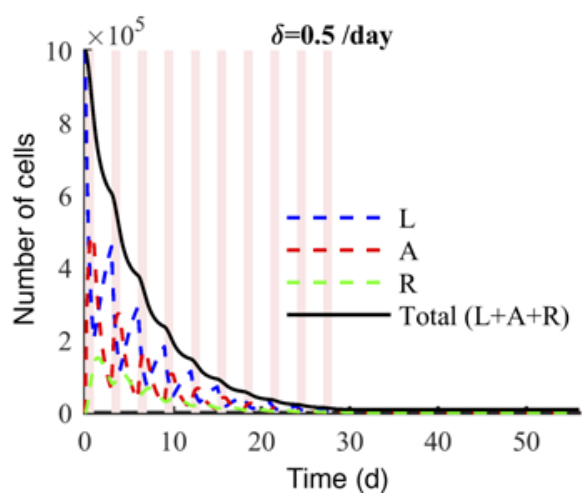

Figure 2. The dynamics of the HIV latent reservoir under single or multiple LRA dosing cycles and the dependence of the LRA efficacy on the induced cell clearance rate. (A) Time course simulations of the mathematical model showing the reservoir dynamics during one LRA dosing cycle, where a patient is treated with LRA for 1 day (shaded in light red) followed by a 2-day resting period. The only difference between the 2 simulations is that $\delta=$ $0.05 /$ day in the simulation on the left and $\delta=0.5 /$ day in the simulation on the right. The uninduced cells $(L)$, the induced cells $(A)$, the refractory cells $(R)$, and the size of latent reservoir $(L+A+R)$ are shown in blue, red, green, and black, respectively. (B) Time course simulations of the reservoir dynamics during and after 10 LRA dosing cycles. In both panel $\mathbf{A}$ and $\mathbf{B}$, increasing the clearance rate from $0.05 /$ day to $0.5 /$ day significantly increases the LRA efficacy. Baseline parameter values used are $\alpha=1.8$ /day on LRA, $\alpha=0 /$ day off LRA, $\gamma=0.5 /$ day, $\omega=1 /$ day, $\rho=0.0072 /$ day, $d=0.006 /$ day,$\eta=0.0017 /$ day (unless varied as indicated on the plots).

increases from 0.05 to 0.5 per day, then the reservoir reduction changes from $5 \%$ to $40 \%$ for one dosing cycle of LRA treatment. This is not surprising because in order to reduce the size of the reservoir, latently infected cells have to be induced and then cleared.

We then considered treatments with multiple dosing cycles. In a clinical trial reported by Archin et al. (11), a patient is given multiple dosing cycles of the LRA vorinostat with approximately 72 hours between each dose (dosing every 3 days). Here, we use our model to evaluate the impact of pulsatile treatment regimens on the latent reservoir. We assume that in a pulsatile treatment, a patient is treated for a total of 10 doses, with the LRA given every third day, as in the protocol selected by Archin et al. (11). Further, we assume that after a dose is given the LRA is active for 1 day and then inactive for the next 2 days. More realistic models could incorporate drug-specific pharmacokinetics and pharmacodynamics. The size of the reservoir is measured during a follow-up at 4 weeks after the last LRA dose as a measure of LRA efficacy. As seen in the 1-dosing-cycle simulations (Figure 2A), we found that with pulsatile treatment increasing the clearance rate induced by the LRA would also substantially increase the LRA efficacy. As shown in Figure 2B, the reservoir size was reduced by $99.2 \%$ when $\delta=0.5 /$ day after 10 dosing cycles compared with $42 \%$ when $\delta=0.05 /$ day.

We further explored how the reduction in the reservoir size depends on variations of the parameters in the model. We found that increasing both the LR rate and the clearance rate would greatly improve 

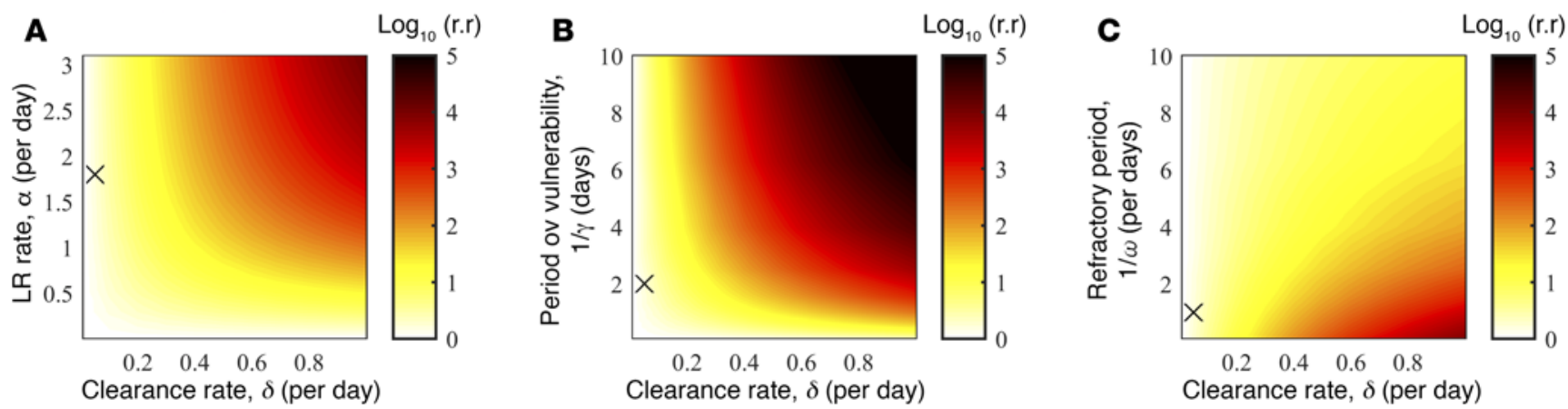

Figure 3. The determinants of LRA efficacy in a 10-dose LRA cycle regimen. (A) The LR rate $(\alpha)$ and the clearance rate $(\delta)$ are equally important in determining LRA efficacy. Color indicates reservoir reduction (r.r.) (in $\log _{10}$ ) at 4-week follow-up after a 10-dose LRA cycle regimen. (B) Increasing the period of vulnerability $(1 / \gamma)$ can substantially increase LRA efficacy when the clearance rate, $\delta$, is high. (C) Decreasing the refractory period (1/ $\omega)$ can further reduce the reservoir size when the clearance rate, $\delta$, is high, although the impact of this parameter on reservoir reduction is not as strong as that of $\alpha, \delta, \gamma$, and $\omega$. Across the 3 panels, ' $x$ ' shows the baseline parameter values used (given in the caption for Figure 2).

LRA efficacy (Figure 3A). Unsurprisingly, the LR rate, $\alpha$, and the clearance rate, $\delta$, are equally important for reservoir reduction, and the highest reduction is achieved when both rates are high, a result consistent with our analytical approximation. With the previously estimated LR and clearance rates $(\alpha=1.8$ /day and $\delta<0.1 /$ day; ' $x$ ' in Figure $3 \mathrm{~A}$ ), a priority would be increasing the clearance rate. For example, if the clearance rate is increased to $0.2,0.5$, or 0.8 /day, there will be approximately a $10-, 100-$, or 1,000-fold reduction, respectively, in the reservoir size after 10 LRA doses.

We then examined the impact of changes in the exit rate $(\gamma)$ and the latency re-establishment rate $(\omega)$ on LRA efficacy (Figure 3B). Note again that $1 / \gamma$ is the period of vulnerability (for immune killing) and $1 / \omega$ is the refractory period of latently infected cells. With the previously estimated LR and clearance rates $(\alpha=$ $1.8 /$ day and $\delta<0.1 /$ day; ' $x$ ' in Figure 3B), increasing the period of vulnerability or decreasing the refractory period will not substantially improve the LRA's efficacy (Figure 3, B and C). However, with an effective LRA (or combinations of LRAs) such that the clearance rate is increased (e.g., to $0.5 /$ day), increasing the period of vulnerability will achieve substantial reservoir reduction (Figure 3B). Decreasing the refractory period can further deplete the reservoir, although its impact is not as strong as increasing the LR rate, the clearance rate, or the period of vulnerability (Figure 3C).

The model predictions above suggest that an obvious priority for developing LRAs or combination therapies currently is focusing on improving HIV antigen expression to increase the ability of the immune response to recognize and clear induced cells. Once an LRA that effectively reduces the size of reservoir is identified, efforts that aim to increase the period of vulnerability can be very useful.

LRA properties that improve efficacy in a pulsatile LRA regimen. As LRAs are directed at host rather than viral targets, side effects and toxicities are likely $(12,46)$. Thus, to achieve a clinically relevant reservoir reduction, multiple LRA exposures where a resting period in between LRA dosing is necessary to avoid side effects, i.e., a pulsatile LRA regimen should be used. We used our model to identify the characteristics of an LRA that can improve efficacy in a pulsatile LRA regimen compared with a continuous LRA regimen (Figure 4). We simulated the model under a 10-dose-cycle pulsatile regimen and compared the results with a continuous 10-day dosing regimen (Figure 4, A and B). Results show surprisingly that when the clearance rate $\delta$ is high and the period of vulnerability is long both on and off the LRA, a pulsatile treatment regimen may reduce the latent reservoir to a lower level than a continuous treatment regimen (Figure 4D). A pulsatile regimen is beneficial because once latently infected cells are induced by an LRA, these cells may remain vulnerable for a long period of time and thus may be effectively cleared despite the rest period in between LRA doses (Figure 4B). We further simulated the model to explore how the reduction in the reservoir size changes with variations in the pulsatile dosing pattern. In general, we found that one LRA dose followed by a sufficiently long resting period before another LRA dose is the best strategy (Supplemental Figure 1), although this result must be taken cautiously because many factors that are not considered in our model may also be important in determining the best treatment strategy, some of which are outlined below.

Essential to the prediction of the benefit of a pulsatile treatment is the assumption that the period of vulnerability continues for some time after LRA dosing. This assumption implies that once an HIV antigen 
A

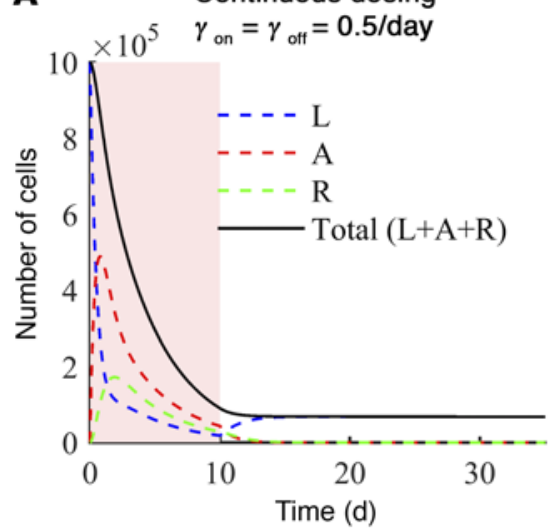

B

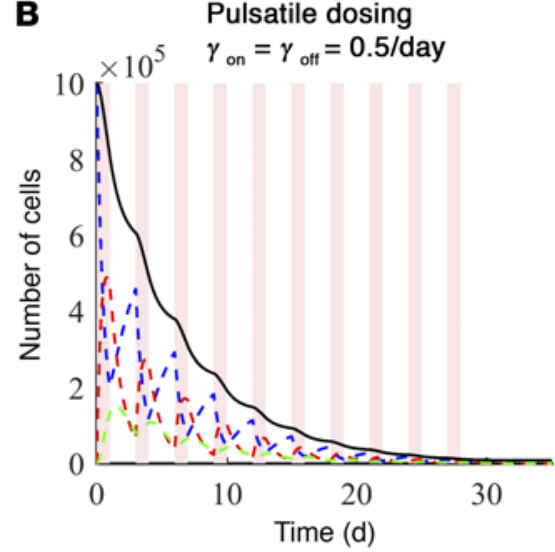

C

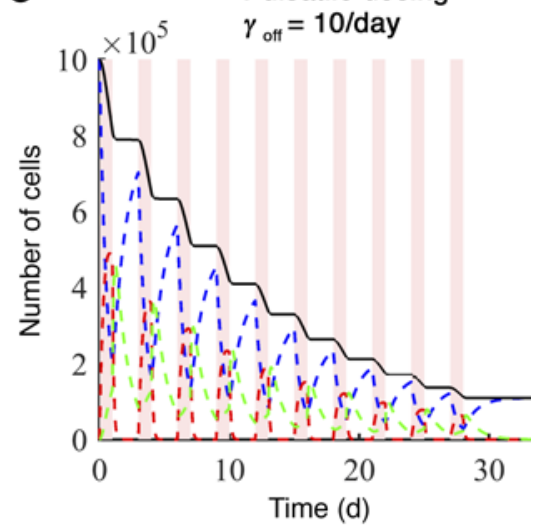

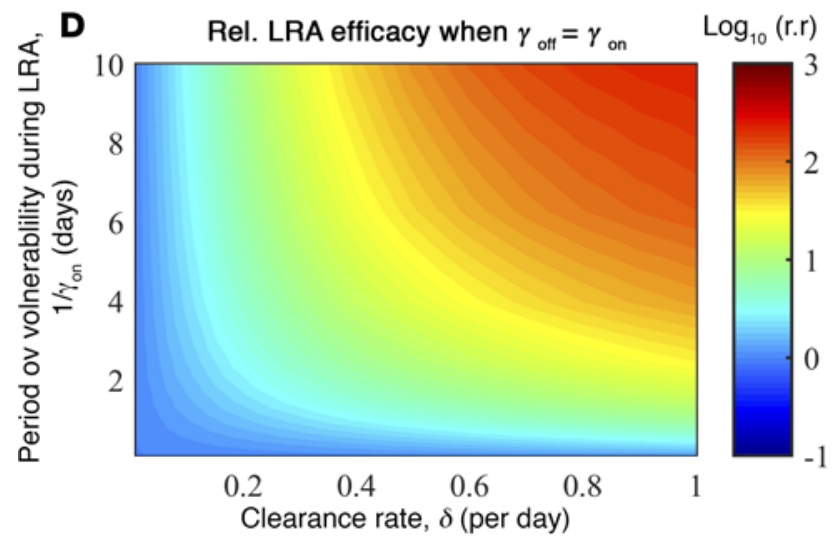

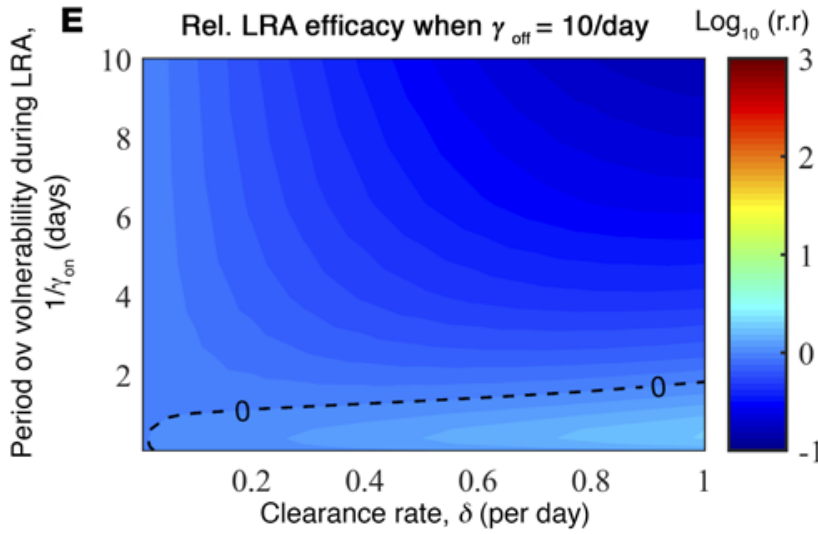

Figure 4. A patient would benefit from a pulsatile regimen if an LRA induces a long period of vulnerability both on and off LRA treatment. (A) Model simulation for a continuous 10-day LRA exposure followed by a rest period. The period of vulnerability is assumed to be 2 days both on and off LRA ( $\gamma_{\text {on }}=\gamma_{\text {off }}$ $=0.5 /$ day). (B) A model simulation for a pulsatile LRA exposure (10 dosing cycles with 1 day on LRA and 2 days off LRA). Same as in panel $\mathbf{A}, \gamma_{\text {on }}=\gamma_{\text {off }}=0.5 /$ day. (C) Same pulsatile LRA exposure as in panel B, except that the period of vulnerability is much shorter, i.e., $\gamma_{\text {off }}=10 /$ day and $1 / \gamma_{\text {off }}=0.1$ day. (D) Comparison of reservoir reduction (r.r.) between a pulsatile exposure and a continuous exposure evaluated 4 weeks after the last dose when $\gamma_{\text {on }}=\gamma_{\text {off: }}$ Color denotes the $\log _{10}$ ratio of the reservoir reduction in a 10-cycle pulsatile LRA exposure over the reduction in a 10-day continuous LRA exposure (followed by a 4-week resting period). (E) Same comparison as in panel $\mathbf{D}$ except that $\gamma_{\text {off }}$ is fixed at $10 /$ day. The dashed contour line denotes parameter combinations where a pulsatile exposure and a continuous exposure achieve equal reservoir reduction. Baseline parameter values are the same as in the caption for Figure 2 .

is expressed on a cell surface, the duration of the antigen expression is long irrespective of the presence of LRA, leading to a long period of vulnerability. This is plausible if after LRA induction, either the duration of HIV gene expression is mostly determined by properties of the HIV gene regulatory circuit (47) or the LRA induces long-lasting changes in the cell state such that sustained HIV gene expression is possible $(13,31)$. Whether this occurs or not is complex, as HIV expression is dependent on many cellular factors, including histone acetylation status (48-51), positive regulators, such as p-TEFb (42-44) and NF- $\mathrm{kB}(52)$, as well as inhibitory molecules, such as Murr1 and human schlafen $11(45,46)$.

Removal of an LRA can lead to changes in expression of host factors $(14,15)$. This may or may not lead to repression of HIV expression (22), depending on the LRA. If HIV expression is dampened and antigen disappears from the cell surface quickly in the absence of LRA, the cells would quickly become invisible to immune effector cells. In this case, the exit rate would be different during LRA and after LRA (which are denoted in the model as $\gamma_{o n}$ and $\gamma_{o f f}$ respectively), and the period of vulnerability would be shortened, i.e., $\gamma_{o f f}>\gamma_{o n}$. Our model results show that a pulsatile treatment regimen may be less effective in reducing the reservoir size compared with a continuous treatment especially when $\gamma_{o f f}>\gamma_{o n}$ and the clearance rate, $\delta$, is high (dark blue region in Figure 4E). In this case, during the holiday periods induced cells mostly reestablish latency (rather than being killed).

Overall, our results suggest that an LRA that induces a long period of vulnerability of latently infected cells both during and after LRA treatment would be more effective in reducing the reservoir size with a pulsatile regimen than with a continuous regimen.

The role of latently infected cell proliferation. Recent work suggests that homeostatic proliferation is an important mechanism that maintains the stability of the latent reservoir $(29,30,53)$. This implies that the stability 

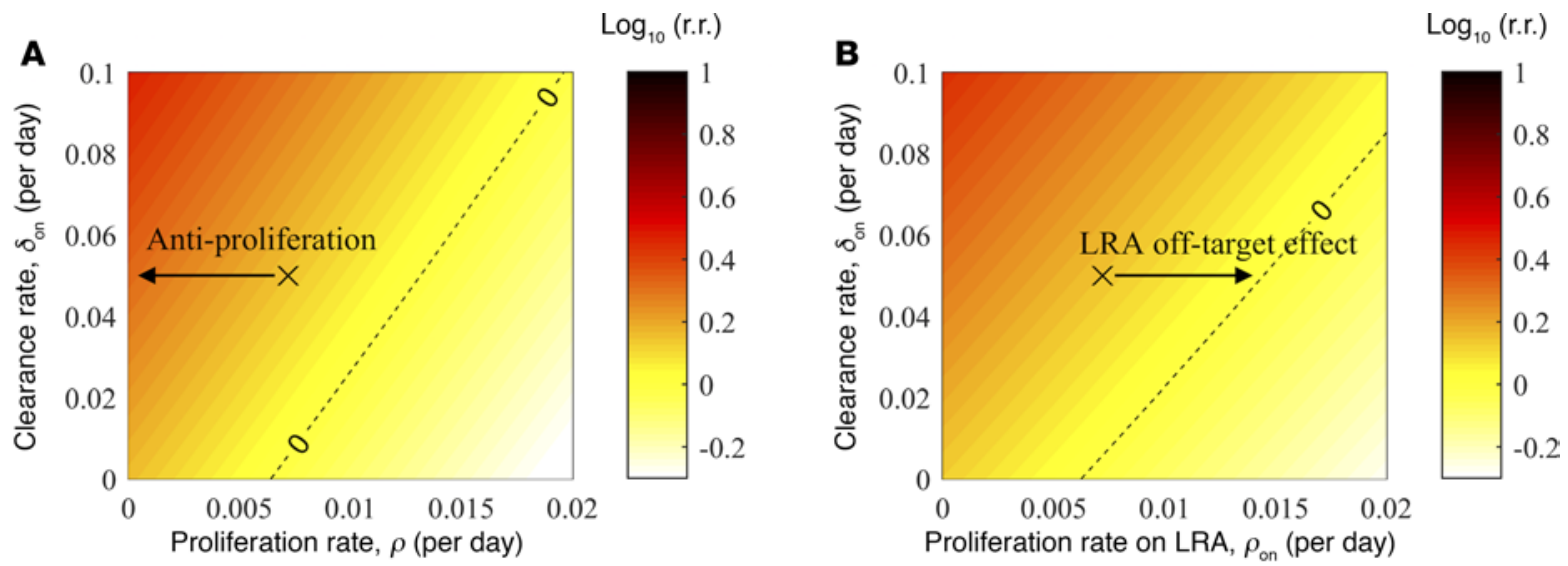

Figure 5. The proliferation rate is an important parameter determining reservoir size. (A) Antiproliferation therapy (left arrow) that reduces the proliferation rate below its baseline value (black vertical dashed line) would enhance LRA treatment and further increase the reservoir reduction (r.r.). The proliferation rate is set at the indicated values both during and after LRA treatment during a 10-dosing cycle as shown in Figure 2B. (B) The proliferation rate is set at the indicated values during LRA treatment only, and it is set to the baseline value 0.0072/day off LRA treatment. If an LRA off-target effect increases the proliferation rate (right arrow), the reservoir size will be reduced to a lesser extent, and the reservoir size may even increase $\left(\log _{10}\right.$ r.r. $\left.<0\right)$ if the increase in the proliferation rate is large enough. Colors denote the $\log _{10}$ reservoir reduction (log $\log _{10}$ r.r.). Across the 2 panels ' $x$ ' shows the baseline parameter values used (given in the caption for Figure 2). The dashed contour lines denote parameter combinations where no reduction in the size of the reservoir is achieved, i.e., $\log _{10}$ r.r. $=0$.

of the latent reservoir is achieved through a balance of proliferation, activation, and death. To investigate the importance of the homeostatic proliferation rate $(\rho)$ in determining LRA efficacy, we examined how changes in this rate affect reservoir reduction. Interventions such as antiproliferative drugs $(35,54)$ have been suggested to reduce the size of the reservoir. Thus, we first focused on the impact of reducing the proliferation rate both in the presence and the absence of an LRA (Figure 5A). A slight decrease in this rate leads to relatively large reduction in the reservoir size (note the small range of variation in $\rho$ we tested in Figure 5A). With the baseline rate of proliferation assumed in our study, i.e., $\rho=0.0072 /$ day, the absolute reservoir reduction is not high even when proliferation is completely inhibited; if the actual proliferation rate is higher than the value we assumed, the absolute reduction can be large. Second, we increased the proliferation rate in the presence of an LRA only, to consider the impact of a potential off-target effect of an LRA on increasing cell proliferation. Again, a slight increase in the latent cell proliferation rate during LRA treatment would compromise the reduction in reservoir size. If the increase is large, it is possible for the reservoir size to increase after LRA treatment (Figure 5B). These results are consistent with the insight from the analytical expressions for $\Psi$ that changes in the proliferation rate can have a large impact on the reservoir size. Overall, our results suggest that the potential off-target effects of LRA administration should be closely examined, and LRAs that do not increase proliferation would be preferred. Although HDACis may not be the ideal LRA, the fact that these agents do not induce proliferation or cell activation was one reason they were initially selected for testing.

\section{Discussion}

Extensive research efforts have focused on developing and testing LRAs to purge the HIV latent reservoir (5, 8). Better methods to evaluate, select, and improve candidate LRAs are needed. Here, we developed a formal quantitative framework integrating essential processes of latency maintenance and reversal to quantify the contribution of each step of latency reversal to the reservoir decline during LRA exposure. We showed that for current LRAs, a priority is to improve those LRAs or to develop combination strategies to increase the clearance of latently infected cells. Once such an improvement is achieved, increasing the period of vulnerability, i.e., the period of HIV and antigen expression, would lead to more rapid reservoir depletion. Interestingly, we found that patients may benefit from a pulsatile treatment regimen when the period of vulnerability is long. Clinical and experimental studies found that LRAs induced HIV expression in latently infected cells (10, $12,13,21)$, suggesting the latency reversal rate $(\alpha)$ may be relatively high. However, the impact of LRAs on the latent cell clearance rate is negligible in vivo, as we and others have shown $(12,13,15,31)$. Unsurprisingly, increasing the clearance rate would substantially increase the LRA efficacy, as suggested in previous studies $(12,13,15,31)$ and consistent with the results presented here. With the current estimate of the LR 
rate, we predict that there will be a 10-, 100-, or 1,000-fold reservoir reduction after 10 LRA doses, if the clearance rate is increased to $0.2,0.5$, or 0.8 /day, respectively. The clearance rate in our model includes immune recognition, binding of and subsequent killing of induced latently infected cells. This (with previous studies; refs. 10, 12, 13, 21) highlights the need to develop LRAs or combination strategies to increase the efficiency of immune recognition and killing of latently infected cells. The clearance of residual HIV infection in the context of prolonged suppression of viral replication is a unique challenge for the immune system. Rare populations of latently infected cells, induced by LRAs to express HIV antigen in limited quantity and for limited time, may be widely distributed across anatomical compartments. In some cases, the HIV-specific immune response may have waned or been depleted by the time of LRA administration. One promising strategy is the combination of LRAs and immunotherapies, for example using checkpoint inhibitors (55) or bispecific antibodies such as the dual-affinity retargeting molecules (56-58). These bispecific antibodies would facilitate recognition and binding between effector cells and latently infected cells expressing HIV antigen. Alternatively, T cell therapeutic vaccines have been suggested to boost the recognition and killing of latently infected cells by $\mathrm{T}$ cells $(59,60)$, although one recent clinical trial failed to show efficacy as measured by the time to viral rebound after ART interruption (61).

Previous studies have focused on the impact of LRAs on the induction of HIV expression and the clearance of latently infected cells $(10-13,15,18,21,22)$. Here, we showed that in addition, a largely overlooked parameter (see ref. 11 for exceptions), the period of vulnerability (i.e., the inverse of the exit rate in our model), which describes the duration of the induced state and HIV antigen expression on the surface of latently infected cells, plays an important role in determining LRA efficacy. The benefits of a longer period of vulnerability come from 2 sources. First, cells expressing HIV antigens for a longer period would have a higher chance of being recognized and cleared by immune effector cells. Second, if the period of vulnerability is long and antigen expression continues after LRA treatment is interrupted, patients may benefit from a pulsatile treatment, as immune recognition and killing of latent cells may continue during the rest periods between LRA doses. Thus, we argue that measurement of the duration of HIV antigen expression on latently infected cells and the efficiency of their recognition by cytotoxic T lymphocytes (62) will be critical for evaluation of LRA candidates. As far as we know this parameter has not been experimentally measured after LRA administration and we hope this work will stimulate attempts to measure this quantity. If the duration of antigen expression correlates with the duration of HIV gene expression, the duration of antigen expression can be estimated indirectly from experiments measuring HIV expression in latently infected cells over time both on and off LRA treatment (see ref. 22 for an example). Our result also suggests that another dimension for optimizing LRA design is to develop LRAs that induce sustained HIV antigen expression on latently infected cells.

Recently, multiple lines of evidence suggest that latently infected cell proliferation is more significant than previously appreciated, and may represent a major mechanism in the maintenance of the latent reservoir $(29,30,53,63)$. Thus, interventions using antiproliferative drugs $(35,54)$ have been proposed to reduce the reservoir size. Our analysis showed the profound impact of variations in the cell proliferation rate on the reservoir size, consistent with a previous analysis (35). For the relatively small proliferation rate assumed in the model, however, we find that the reduction in the reservoir size is relatively mild, and to achieve substantial reduction in the reservoir size within a short period of time, an effective LRA is needed. However, if the proliferation rate is much higher than that used in the study, antiproliferative agents may be effective in reducing the size of the latent reservoir $(30,35)$. Thus, an important direction for future work is to estimate the rate of latently infected cell proliferation and the extent to which proliferation contributes to the maintenance of the reservoir.

The model presented here integrates many key aspects of latency reversal under LRA treatment. Inevitably, the model makes many simplifying assumptions, as the biology of latency reversal (7) is complex. First, we assumed that the latently infected cell population is a homogeneous population. However, latently infected cells consist of different types of cells, such as central memory $\mathrm{T}$ cells, effector memory cells, and transitional memory T cells (29). These latently infected cell populations may reside in different tissue compartments with different dynamics in the presence of an LRA. Further, in addition to the heterogeneity in cell types, it has been shown that the replication-competent proviruses in the latently infected cells are heterogeneous in their CTL epitopes, and these differences may lead to different clearance rates of infected cells (64). Therefore, model extensions with multiple strains and multiple compartments accounting for different types of latently infected cells and latent viruses in different tissue 
compartments may be useful. However, the lack of measurements of latent infection in heterogeneous cell populations in different tissue compartments limits the predictability of such complex models. The general conclusions of our model can be viewed as a mean field approximation to these more complicated situations. Second, without losing generality, we have assumed that the clearance rate of induced cells is independent of the presence or absence of LRAs. This may be a good assumption for most LRAs but experimental studies have revealed exceptions $(65,66)$. Our model can be easily adapted to the individual characteristics of LRAs. Third, we used a step function to describe the impact of an LRA, i.e., the LR parameter, $\alpha$, changes from 0 to a constant value upon LRA treatment. A model incorporating the pharmacokinetics and pharmacodynamics of LRAs will be useful to evaluate how drug characteristics, such as their $\mathrm{EC}_{50}$ and the slope of the dose-response curve (67-69), impacts its efficacy. Last, another extension of the model is to incorporate LRA side effects into the model and explicitly describe how side effects depend on the pharmacokinetics of the LRA. This type of model will be useful to make precise predictions about the best way to pulse a treatment regimen, although currently the lack of data again prevents such model extension. Even with all simplifying assumptions made here, the simple model we developed sheds light on the contribution of each of the steps in the latency reversal and clearance strategy towards the reduction of the HIV reservoir, such that general principles for determining LRA efficacy, and thus optimizing LRA combinations and treatment strategies, can be made.

Overall, we highlight the importance of developing LRAs to induce sustained HIV antigen expression to maximize the opportunity for persistently infected cells to become vulnerable to effector mechanisms, and the need for augmentation of immune effector mechanisms to clear these targets. The model framework we presented here serves as a useful tool to evaluate the efficacy of and derive design principles for LRAs and combination strategies using immunotherapies $(7,56,58)$ or other approaches to clear persistent HIV infection.

\section{Methods}

The mathematical model. In our model we keep track of 3 types of latently infected cell populations: uninduced latently infected cells $(L)$, cells induced by an LRA $(A)$, and cells that have become refractory to LRA stimulation following induction $(R)$. The ODEs describing these cell populations are:

$$
\begin{aligned}
& d L / d t=(\rho-d) L-\eta L-\alpha L+\omega R \\
& d A / d t=(\rho-\delta) A+\alpha L-\gamma A \\
& d R / d t=(\rho-d) R+\gamma A-\omega R
\end{aligned}
$$

In this model (see Figure 1 for a schematic diagram), latently infected cells $(L, A$, and $R$ ) all proliferate at per capita rate $\rho$. In the absence of LRA, uninduced latently infected cells $(L)$ die at per capita rate $d$, and are naturally activated to become productively infected cells (70) (a different population from the cells induced by an LRA) at per capita rate $\eta$. Throughout this study, we assume that patients are treated with effective cART and the contribution of viruses produced from productively infected cells and induced cells to further rounds of infection and to the latent reservoir are neglected $(31,43)$. Therefore, in our model, latently infected cells are maintained by proliferation. Although one study proposed on-going viral replication in CART sanctuaries as another major mechanism for reservoir maintenance (71), other studies suggest that on-going viral replication is inconsistent with a wide range of clinical observations (72-74).

LRA treatment turns uninduced cells into induced cells $(A)$ at rate $\alpha$ (the LR rate), where $\alpha>0$ in the presence of an LRA and $\alpha=0$ in the absence of an LRA. Once induced, we assume that latently infected cells produce HIV RNAs and proteins and consequently express HIV antigens. HIV antigens may be recognized by the immune system and the cells expressing these antigens are assumed to be killed by immune effector cells at per capita death rate $\delta$ (the clearance rate). If induced cells are not recognized by the immune system after a certain period, HIV gene expression declines, leading to reestablishment of latency (instead of death) (22) and loss of HIV antigens from the cell surface due to lack of production and continuing membrane turnover (75). In our model, we assume for simplicity that the process from loss of HIV gene expression to removal of HIV antigen from the cell surface to the extent that the cell becomes invisible to the immune system occurs at rate $\gamma$ (we term the 'exit rate'); cells are then in the refractory stage $(R)$. Therefore, the period during which the cell is visible to the immune response, i.e., the period of 'vulnerability' termed in other studies $(11,76)$, can be calculated as $1 / \gamma$. Note that, in our analyses, we 
considered the case when the period of vulnerability is different depending on whether an LRA is present, and the period is denoted as $1 / \gamma_{\text {on }}$ and $1 / \gamma_{\text {off }}$ in the presence and absence of an LRA, respectively. We assume that the cells leave the refractory state and revert to uninduced cells $(L)$ at rate $\omega$, i.e., the cells stay in the refractory state $(R)$ for an average period of $1 / \omega$.

Parameter values. We set the death rate for uninduced cells and refractory cells, $d$, to $0.006 /$ day, since latently infected cells are mostly resting memory $\mathrm{CD} 4^{+} \mathrm{T}$ cells, which have an estimated mean lifetime of approximately 164 days $(77,78)$. We set the natural activation rate, $\eta$, to be $0.0017 /$ day as previously reported (43). The rate $\rho$ is set to 0.0072 /day such that in the absence of LRA, the half-life of latent reservoir is 44 months $(2,3)$. Note that precise quantification of these parameter values is lacking; other parameter choices could also be made that would be consistent with the observed 44-month latent reservoir half-life. The predictions of the model do not strongly depend on the choice of the death rate $d$ and the natural activation rate $\eta$, as long as the half-life of the reservoir size is set to 44 months, because these rates are relatively low compared with the clinically relevant timeframe of LRA exposure that we study.

\section{Author contributions}

RK, DMM, and ASP designed the research study. RK, JMC, and ASP conducted model construction and analyses, RK, JMC, DMM, and ASP wrote the manuscript.

\section{Acknowledgments}

Portions of this work were performed under the auspices of the US Department of Energy under contract DE-AC52-06NA25396 and supported by NIH grants R01-OD011095, R01-AI028433, and P01-AI131365 (to ASP), U01-AI17844 and UM1-AI126619 (to DMM), and the Collaboratory of AIDS Researchers for Eradication, the UNC CFAR Developmental Award-UNC CFAR P30 AI50410 (to RK), and by the National Science Foundation under grant DMS-1714654 (to JMC).

Address correspondence to: Alan S. Perelson, Theoretical Biology \& Biophysics, Mail Stop K710, Los Alamos National Laboratory, Los Alamos, New Mexico 87545, USA. Phone: 505.667.6829; Email: asp@lanl.gov.

1. Antiretroviral Therapy Cohort Collaboration. Survival of HIV-positive patients starting antiretroviral therapy between 1996 and 2013: a collaborative analysis of cohort studies. Lancet HIV. 2017;4(8):e349-e356.

2. Crooks AM, et al. Precise quantitation of the latent HIV-1 reservoir: Implications for eradication strategies. $J$ Infect Dis. 2015;212(9):1361-1365.

3. Siliciano JD, et al. Long-term follow-up studies confirm the stability of the latent reservoir for HIV-1 in resting CD4 ${ }^{+} \mathrm{T}$ cells Nat Med. 2003;9(6):727-728.

4. Deeks SG. HIV: Shock and kill. Nature. 2012;487(7408):439-440.

5. International AIDS Society Scientific Working Group on HIV Cure, et al. Towards an HIV cure: a global scientific strategy. Nat Rev Immunol. 2012;12(8):607-614.

6. Margolis DM, Archin NM. Proviral latency, persistent human immunodeficiency virus infection, and the development of latency reversing agents. JInfect Dis. 2017;215(supp1_3):S111-S118.

7. Margolis DM, Garcia JV, Hazuda DJ, Haynes BF. Latency reversal and viral clearance to cure HIV-1. Science. 2016;353(6297):aaf6517.

8. Richman DD, Margolis DM, Delaney M, Greene WC, Hazuda D, Pomerantz RJ. The challenge of finding a cure for HIV infection. Science. 2009;323(5919):1304-1307.

9. Rasmussen TA, Lewin SR. Shocking HIV out of hiding: where are we with clinical trials of latency reversing agents? Curr Opin HIV AIDS. 2016;11(4):394-401.

10. Archin NM, et al. HIV-1 expression within resting $\mathrm{CD}^{+} \mathrm{T}$ cells after multiple doses of vorinostat. J Infect Dis. 2014;210(5):728-735.

11. Archin NM, et al. Interval dosing with the HDAC inhibitor vorinostat effectively reverses HIV latency. J Clin Invest. 2017;127(8):3126-3135.

12. Archin NM, et al. Administration of vorinostat disrupts HIV-1 latency in patients on antiretroviral therapy. Nature. 2012;487(7408):482-485.

13. Elliott JH, et al. Activation of HIV transcription with short-course vorinostat in HIV-infected patients on suppressive antiretroviral therapy. PLoS Pathog. 2014;10(10):e1004473.

14. Søgaard OS, et al. The depsipeptide romidepsin reverses HIV-1 latency in vivo. PLoS Pathog. 2015;11(9):e1005142.

15. Rasmussen TA, et al. Panobinostat, a histone deacetylase inhibitor, for latent-virus reactivation in HIV-infected patients on suppressive antiretroviral therapy: a phase 1/2, single group, clinical trial. Lancet HIV. 2014;1(1):e13-e21.

16. Bouchat $\mathrm{S}$, et al. Histone methyltransferase inhibitors induce HIV-1 recovery in resting CD4(+) T cells from HIV-1-infected HAART-treated patients. AIDS. 2012;26(12):1473-1482.

17. Spivak AM, et al. A pilot study assessing the safety and latency-reversing activity of disulfiram in HIV-1-infected adults on 
antiretroviral therapy. Clin Infect Dis. 2014;58(6):883-890.

18. Xing S, et al. Disulfiram reactivates latent HIV-1 in a Bcl-2-transduced primary CD4 ${ }^{+} \mathrm{T}$ cell model without inducing global T cell activation. J Virol. 2011;85(12):6060-6064.

19. Darcis G, et al. An in-depth comparison of latency-reversing agent combinations in various in vitro and ex vivo HIV-1 latency models identified bryostatin-1+JQ1 and ingenol-B+JQ1 to potently reactivate viral gene expression. PLoS Pathog. 2015;11(7):e1005063.

20. Banerjee C, et al. BET bromodomain inhibition as a novel strategy for reactivation of HIV-1. J Leukoc Biol. 2012;92(6):1147-1154

21. Laird GM, et al. Ex vivo analysis identifies effective HIV-1 latency-reversing drug combinations. J Clin Invest. 2015;125(5):1901-1912.

22. Shan L, Xing S, Yang HC, Zhang H, Margolick JB, Siliciano RF. Unique characteristics of histone deacetylase inhibitors in reactivation of latent HIV-1 in Bcl-2-transduced primary resting CD4+ T cells. J Antimicrob Chemother. 2014;69(1):28-33

23. Wu G, et al. HDAC inhibition induces HIV-1 protein and enables immune-based clearance following latency reversal. JCI Insight. 2017;2(16):e92901.

24. Blankson JN, et al. Biphasic decay of latently infected $\mathrm{CD} 4^{+} \mathrm{T}$ cells in acute human immunodeficiency virus type 1 infection. $J$ Infect Dis. 2000;182(6):1636-1642.

25. Chun TW, Finzi D, Margolick J, Chadwick K, Schwartz D, Siliciano RF. In vivo fate of HIV-1-infected T cells: quantitative analysis of the transition to stable latency. Nat Med. 1995;1(12):1284-1290.

26. Eriksson S, et al. Comparative analysis of measures of viral reservoirs in HIV-1 eradication studies. PLoS Pathog. 2013;9(2):e1003174

27. Lee SK, et al. Quantification of the latent HIV-1 reservoir using ultra deep sequencing and primer ID in a viral outgrowth assay. J Acquir Immune Defic Syndr. 2017;74(2):221-228.

28. Lorenzi JC, et al. Paired quantitative and qualitative assessment of the replication-competent HIV-1 reservoir and comparison with integrated proviral DNA. Proc Natl Acad Sci USA. 2016;113(49):E7908-E7916.

29. Chomont N, et al. HIV reservoir size and persistence are driven by T cell survival and homeostatic proliferation. Nat Med. 2009;15(8):893-900

30. Hosmane NN, et al. Proliferation of latently infected CD4 $4^{+} \mathrm{T}$ cells carrying replication-competent HIV-1: Potential role in latent reservoir dynamics. J Exp Med. 2017;214(4):959-972.

31. Ke R, Lewin SR, Elliott JH, Perelson AS. Modeling the effects of vorinostat in vivo reveals both transient and delayed HIV transcriptional activation and minimal killing of latently infected cells. PLoS Pathog. 2015;11(10):e1005237.

32. Petravic J, Rasmussen TA, Lewin SR, Kent SJ, Davenport MP. Relationship between measures of HIV reactivation and decline of the latent reservoir under latency-reversing agents. J Virol. 2017;91(9):e02092-16.

33. Conway JM, Coombs D. A stochastic model of latently infected cell reactivation and viral blip generation in treated HIV patients. PLoS Comput Biol. 2011;7(4):e1002033.

34. Petravic J, Martyushev A, Reece JC, Kent SJ, Davenport MP. Modeling the timing of antilatency drug administration during HIV treatment. J Virol. 2014;88(24):14050-14056.

35. Reeves DB, Duke ER, Hughes SM, Prlic M, Hladik F, Schiffer JT. Anti-proliferative therapy for HIV cure: a compound interest approach. Sci Rep. 2017;7(1):4011.

36. Hill AL, Rosenbloom DI, Fu F, Nowak MA, Siliciano RF. Predicting the outcomes of treatment to eradicate the latent reservoir for HIV-1. Proc Natl Acad Sci USA. 2014;111(37):13475-13480.

37. Ho DD, Neumann AU, Perelson AS, Chen W, Leonard JM, Markowitz M. Rapid turnover of plasma virions and CD4 lymphocytes in HIV-1 infection. Nature. 1995;373(6510):123-126.

38. Perelson AS, et al. Decay characteristics of HIV-1-infected compartments during combination therapy. Nature. 1997;387(6629):188-191.

39. Perelson AS. Modelling viral and immune system dynamics. Nat Rev Immunol. 2002;2(1):28-36.

40. Louie M, et al. Determining the relative efficacy of highly active antiretroviral therapy. J Infect Dis. 2003;187(6):896-900

41. Perelson AS, Neumann AU, Markowitz M, Leonard JM, Ho DD. HIV-1 dynamics in vivo: virion clearance rate, infected cell life-span, and viral generation time. Science. 1996;271(5255):1582-1586.

42. Cardozo EF, Andrade A, Mellors JW, Kuritzkes DR, Perelson AS, Ribeiro RM. Treatment with integrase inhibitor suggests a new interpretation of HIV RNA decay curves that reveals a subset of cells with slow integration. PLoS Pathog. 2017;13(7):e1006478.

43. Conway JM, Perelson AS. Residual viremia in treated HIV+ individuals. PLoS Comput Biol. 2016;12(1):e1004677.

44. Reardon B, et al. Dose-responsive gene expression in suberoylanilide hydroxamic acid-treated resting CD $4^{+} \mathrm{T}$ cells. AIDS $2015 ; 29(17): 2235-2244$

45. Halsall JA, Turan N, Wiersma M, Turner BM. Cells adapt to the epigenomic disruption caused by histone deacetylase inhibitors through a coordinated, chromatin-mediated transcriptional response. Epigenetics Chromatin. 2015;8:29.

46. Bratland A, Dueland S, Hollywood D, Flatmark K, Ree AH. Gastrointestinal toxicity of vorinostat: reanalysis of phase 1 study results with emphasis on dose-volume effects of pelvic radiotherapy. Radiat Oncol. 2011;6:33.

47. Weinberger LS, Dar RD, Simpson ML. Transient-mediated fate determination in a transcriptional circuit of HIV. Nat Genet. 2008;40(4):466-470

48. Lusic M, Marcello A, Cereseto A, Giacca M. Regulation of HIV-1 gene expression by histone acetylation and factor recruitment at the LTR promoter. EMBO J. 2003;22(24):6550-6561.

49. He G, Margolis DM. Counterregulation of chromatin deacetylation and histone deacetylase occupancy at the integrated promoter of human immunodeficiency virus type 1 (HIV-1) by the HIV-1 repressor YY1 and HIV-1 activator Tat. Mol Cell Biol. 2002;22(9):2965-2973.

50. Romerio F, Gabriel MN, Margolis DM. Repression of human immunodeficiency virus type 1 through the novel cooperation of human factors YY1 and LSF. J Virol. 1997;71(12):9375-9382.

51. Van Lint C, Emiliani S, Ott M, Verdin E. Transcriptional activation and chromatin remodeling of the HIV-1 promoter in response to histone acetylation. EMBO J. 1996;15(5):1112-1120.

52. Siekevitz M, Josephs SF, Dukovich M, Peffer N, Wong-Staal F, Greene WC. Activation of the HIV-1 LTR by T cell mitogens 
and the trans-activator protein of HTLV-I. Science. 1987;238(4833):1575-1578.

53. Maldarelli F, et al. HIV latency. Specific HIV integration sites are linked to clonal expansion and persistence of infected cells. Science. 2014;345(6193):179-183.

54. Gavegnano C, et al. Novel mechanisms to inhibit HIV reservoir seeding using Jak inhibitors. PLoS Pathog. 2017;13(12):e1006740.

55. Guihot A, et al. Drastic decrease of the HIV reservoir in a patient treated with nivolumab for lung cancer. Ann Oncol. 2018;29(2):517-518.

56. Margolis DM, Koup RA, Ferrari G. HIV antibodies for treatment of HIV infection. Immunol Rev. 2017;275(1):313-323.

57. Sung JA, et al. Dual-affinity re-targeting proteins direct T cell-mediated cytolysis of latently HIV-infected cells. J Clin Invest. 2015;125(11):4077-4090.

58. Ferrari G, Haynes BF, Koenig S, Nordstrom JL, Margolis DM, Tomaras GD. Envelope-specific antibodies and antibody-derived molecules for treating and curing HIV infection. Nat Rev Drug Discov. 2016;15(12):823-834.

59. Sung JA, et al. Expanded cytotoxic T-cell lymphocytes target the latent HIV reservoir. J Infect Dis. 2015;212(2):258-263.

60. Mothe B, et al. Viral control induced by HIVCONSV vaccines \& romidepsin in early treated individuals. Abstract 119LB, CROI2017 abstract eBook, p47, http://www.croiconference.org/sites/default/files/uploads/croi2017-abstract-eBook.pdf.

61. Sneller MC, et al. A randomized controlled safety/efficacy trial of therapeutic vaccination in HIV-infected individuals who initiated antiretroviral therapy early in infection. Sci Transl Med. 2017;9(419):eaan8848.

62. Jones RB, et al. A subset of latency-reversing agents expose HIV-infected resting CD4 ${ }^{+}$T-cells to recognition by cytotoxic T-lymphocytes. PLoS Pathog. 2016;12(4):e1005545.

63. Wagner TA, et al. HIV latency. Proliferation of cells with HIV integrated into cancer genes contributes to persistent infection. Science. 2014;345(6196):570-573.

64. Deng K, et al. Broad CTL response is required to clear latent HIV-1 due to dominance of escape mutations. Nature. 2015;517(7534):381-385.

65. Clutton G, et al. The differential short- and long-term effects of HIV-1 latency-reversing agents on T cell function. Sci Rep. 2016;6:30749.

66. Walker-Sperling VE, Pohlmeyer CW, Tarwater PM, Blankson JN. The effect of latency reversal agents on primary CD8 ${ }^{+} \mathrm{T}$ cells Implications for shock and kill strategies for human immunodeficiency virus eradication. EBioMedicine. 2016;8:217-229.

67. Rosenbloom DI, Hill AL, Rabi SA, Siliciano RF, Nowak MA. Antiretroviral dynamics determines HIV evolution and predicts therapy outcome. Nat Med. 2012;18(9):1378-1385.

68. Sampah ME, Shen L, Jilek BL, Siliciano RF. Dose-response curve slope is a missing dimension in the analysis of HIV-1 drug resistance. Proc Natl Acad Sci USA. 2011;108(18):7613-7618.

69. Jilek BL, et al. A quantitative basis for antiretroviral therapy for HIV-1 infection. Nat Med. 2012;18(3):446-451.

70. Tobin NH, et al. Evidence that low-level viremias during effective highly active antiretroviral therapy result from two processes: expression of archival virus and replication of virus. J Virol. 2005;79(15):9625-9634

71. Lorenzo-Redondo R, et al. Persistent HIV-1 replication maintains the tissue reservoir during therapy. Nature. 2016;530(7588):51-56

72. Kearney MF, et al. Ongoing HIV Replication During ART Reconsidered. Open Forum Infect Dis. 2017;4(3):ofx173.

73. Rosenbloom DIS, Hill AL, Laskey SB, Siliciano RF. Re-evaluating evolution in the HIV reservoir. Nature. 2017;551(7681):E6-E9.

74. van Zyl G, Bale MJ, Kearney MF. HIV evolution and diversity in ART-treated patients. Retrovirology. 2018;15(1):14.

75. Howarth M, Williams A, Tolstrup AB, Elliott T. Tapasin enhances MHC class I peptide presentation according to peptide halflife. Proc Natl Acad Sci USA. 2004;101(32):11737-11742.

76. Sung JA, et al. Vorinostat renders the replication-competent latent reservoir of human immunodeficiency virus (HIV) vulnerable to clearance by CD8 T cells. EBioMedicine. 2017;23:52-58.

77. Westera L, et al. Closing the gap between T-cell life span estimates from stable isotope-labeling studies in mice and humans Blood. 2013;122(13):2205-2212.

78. De Boer RJ, Perelson AS. Quantifying T lymphocyte turnover. J Theor Biol. 2013;327:45-87. 